\title{
Guillain-Barré Syndrome Masquerading As Cervical Myelopathy
}

\author{
Uzzwal Kumar Mallick$^{1}$, Badrul Alam², Mohammad Asaduzzaman ${ }^{3}$
}

\begin{abstract}
Guillain-Barré syndrome (GBS) is an acute inflammatory demyelinating polyneuropathy. In typical cases, the first symptoms of GBS are pain, numbness, paresthesia, weakness in the limbs. Autonomic involvement is common and causes urinary retention and ileus. Most of these symptoms overlap with those of cervical myelopathy. Therefore, correct diagnosis of GBS in a patient with symptomatic cervical myelopathy or in a patient with atypical manifestations of GBS can be difficult, especially early in the course of GBS.

We report a 60-year-old man who was admitted to the neurosurgery department with worsening neck pain, numbness and weakness in the hands initially thought to be secondary to progressive cervical myelopathy. However, his symptoms rapidly progressed to flaccid areflexic quadriparesis and respiratory difficulty within few days and shifted to ICU for ventilator support. Electrophysiological studies and cerebrospinal fluid analysis were consistent with an acquired demyelinating polyradiculoneuropathy. We planned for immunotherapy with intravenous immunoglobulin, but his condition was improving day by day with conservative treatment, so immunotherapy with intravenous immunoglobulin was not initiated. Any patient presenting as unexplain Cervical myelopathy, GBS should be kept in mind before planning any surgical intervention.
\end{abstract}

Keywords: Guillain-Barré syndrome (GBS); cervical myelopathy, Nerve conduction study (NCS)

\section{Introduction:}

Guillain-Barré syndrome (GBS)-Acute Inflammatory Demyelinating Polyradiculoneuropathy, (AIDP) is an acute polyneuropathy that commonly manifests with areflexic flaccid paralysis associated with variable sensory and autonomic disturbances. ${ }^{1}$ Albuminocytologic dissociation; elevated cerebrospinal fluid (CSF) protein without pleocytosis is a typical finding in GBS. ${ }^{1,2}$ GBS is a neurological emergency that affects 1:100,000 person-years in the Western World . ${ }^{1,3}$ Symptoms progress over a period of up to 4 weeks. During and after the acute phase of GBS, the prognosis is extremely variable, nearly $25 \%$ of patients develop respiratory impairment, $20 \%$ remain severely disabled, and 5\% may die, despite immunotherapy ${ }^{1}$.

1. Dr. Uzzwal Kumar Mallick, Registrar, Dept. of Critical Care Medicine, National Institute of Neurosciences and Hospital, Dhaka.

2. Dr. Badrul Alam, Professor of neurology, National Institute of Neurosciences and Hospital, Dhaka.

3. Dr. Mohammad Asaduzzaman, Assistant registrar, Dept. of CCM, National Institute of Neurosciences and Hospital, Dhaka

All authors had equal contribution in writing the manuscript.

\section{Corresponding Author:}

Dr. Uzzwal Kumar Mallick

Registrar

Dept. of Critical Care Medicine

National Institute of Neurosciences and Hospital

Dhaka, Bangladesh

Cell: 01712715180

E-mail: ukm1980@gmail.com.
Cervical myelopathy is one of the most common causes of spinal cord dysfunction in older persons ${ }^{4}$. The aging process results in degenerative changes in the cervical spine that in advanced stages can cause compression of the spinal cord ${ }^{4}$. On the other hand, GBS and Cervical myelopathy both may present as subacute progressive paraparesis or quadriparesis ${ }^{5}$. Patients with GBS typically have reduced or absent reflexes and may have cranial nerve involvement ${ }^{1}$. While patients with cervical myelopathy are usually present with neck and arm pain with weakness and hyperreflexia and do not have cranial nerve involvement ${ }^{4}$. However, these clinical signs could be absent early at the time of the presentation. Therefore, neuroimaging and electrophysiological studies are often required in solving the diagnostic complexity ${ }^{5}$.

Here, we present a case to report of GBS in a patient with chronic cervical myelopathy. We encourage detailed neurologic assessment in patients with cervical myelopathy especially in atypical presentation before consideration for surgical intervention.

\section{CASE REPORT}

A 60-year-old man, hypertensive, shop-keeper, admitted in the neuro-surgery department in National Institute of Neurosciences and Hospital, Dhaka, with the complaints of neck pain for five months. There was no history of bulbar dysfunction, sphincter abnormality, or autonomic symptoms. He had no history of preceding upper respiratory tract or gastrointestinal infection. Neurological examination revealed no cranial nerve dysfunction or generalized hypotonia. 
Plantar responses were flexor on both sides, sensory and cerebellar examinations were normal.

Cervical spine MRI showed multilevel degenerative changes with moderate cord compression, most prominent at the C4-C5 level (Figure 1). The initial working diagnosis was Cervical myelopathy with subacute worsening (compression of the spinal cord). On the 4th day of admission, in neurosurgery dept. the patient's condition became progressively worse; he developed moderate flaccid quadriplegia (power grade 3/5), absent deep tendon reflexes, and swallowing difficulty.

Gradually he developed breathing difficulty and he was transferred to the intensive care unit (ICU).

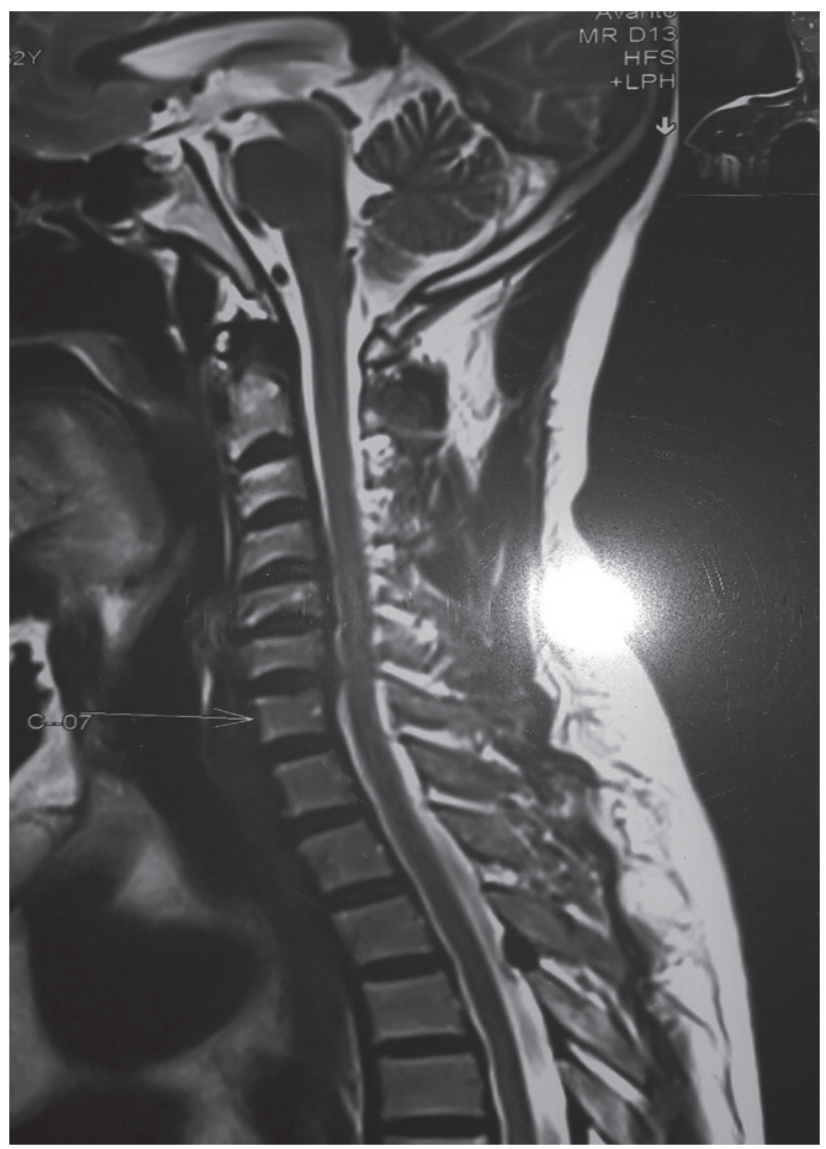

Fig-1: Shows the non-compressive lesion of the cervical cord with signal change on $\mathrm{C} 5 / 6$.

Two days after admission in ICU his respiratory function further deteriorated. ABG (arterial blood gas) showed type-II respiratory failure, then endotracheal intubation was done and patient was kept on mechanical ventilation. During this time Nerve conduction study (NCS) was done and it showed AIDP variety. CSF report showed protein $60 \mathrm{mg} / \mathrm{dl}$, Glucose 3.42 mmol/L, Cells 4/HPF. He was planned to have immunotherapy with intravenous immunoglobulin (IVIG) but from the 7th day of ICU admission, the patient's condition was gradually improving. After weaning we did tracheostomy on 11th day of mechanical ventilation. He was transferred to the HDU on next day. After seven days of staying in HDU, he showed marked improvement and was able to swallow normally, and his muscle strength improved both in the upper and lower extremities. He was discharged home with closure of tracheostomy tube. His hematological and routine metabolic workup were normal. He was discharged in a stable condition, and was advised to get daily physiotherapy. He was advised to come for follow-up for myelopathy. After six months he came for follow-up and we found that he fully recovered and he could walk independently without any helping aid.

\section{Discussion}

Acute myelopathy due to spinal cord compression can be clinically confused with GBS since the deep tendon reflexes may be depressed in these condition ${ }^{4}$. However, early bowel and bladder dysfunction and a sensory level point to myelopathy that is usually supported by finding a focal lesion in the MRI of the spine ${ }^{4,5}$.

When our patient was admitted to the neurosurgery department, he had neck pain and numbness in the fingers that were thought to be due to the progression of cervical myelopathy. Neck pain is not common symptoms of GBS; it is more prevalent in cervical myelopathy. However, neck pain has been rarely reported in the pharyngeal-cervical-brachial variant, which accounts for approximately $3 \%$ of total GBS cases $^{2}$. Our case may represent this variant that would also explain why he developed bulbar weakness soon after admission. Alternatively, his disease could have started with axial and neck hypotonia that had aggravated her preexisting cervical roots compression. The rapid progression of her upper limbs weakness followed by acute quadriplegia and arreflexia high suspicion of AIDP.

The diagnostic challenge, in this case, is that his cervical MRI was abnormal when he attended the ED, showing cervical myelopathy confirmed by signal changes within the cervical spinal cord at the C5 level (Fig-1). In this case, the diagnosis of GBS was confirmed by the electrophysiological study that demonstrated slowing of the nerve conduction velocities, partial motor conduction block that are not typical findings of cervical myelopathy ${ }^{6}$. High protein level in CSF may be observed early in GBS course, although, protein level may not yet be prominent until 1-2 weeks after the onset of weakness, rarely they do remain persistently normal ${ }^{1,2}$. In this case, we diagnosed acute polyradiculoneuropathy superimposing a chronic cervical myelopathy. In reviewing the literature, we found no association between GBS and cervical myelopathy. Only one case similar to our case was reported previously by Abai et al., a 39-year-old lady developed progressive lower and upper limbs weakness and her MRI neck demonstrated cervical spondylosis with cord compression at C5/C6 $6^{5}$. The diagnosis was confirmed by electrophysiological studies. The authors assumed that the patient developed GBS followed by cervical myelopathy while our case is known to have cervical myelopathy followed by GBS. Considering this case and the previously reported one, variable development of symptoms of GBS may coexist in a patient with cervical myelopathy 
highlighting the importance of making appropriate diagnosis especially in patients considered for surgery.

\section{Conclusion}

GBS and cervical myelopathy may coexist. The clinicians should suspect GBS in a patient with cervical myelopathy who presents with symptoms like neck pain, numbness and weakness in the hands with diminish or loss of reflexes that cannot be explained by cervical cord compression alone.

\section{Conflict of Interest}

None

\section{Consent for Manuscript and Figure}

The patient and his wife gave written consent for the use of personal and medical information for the publication of this case report and accompanying images.

\section{References:}

1. Yuki N, Hartung HP (2012) Guillain-Barré syndrome. N Engl J Med 366: 2294-304.

2. Dimachkie MM, Barohn RJ (2013) Guillain-Barré syndrome and variants. NeurolClin 31: 491-510.

3. Alter M (1990) The epidemiology of Guillain-Barré syndrome. Ann Neurol 27: S7-12.

4. Wu JC, Ko CC, Yen YS, Huang WC, Chen YC, et al. (2013) Epidemiology of cervical spondylotic myelopathy and its risk of causing spinal cord injury: a national cohort study. Neurosurg Focus 35: E10.

5. Abai S, Kim SB, Kim JP, Lim YJ (2010) Guillain-barré syndrome combined with acute cervical myelopathy. J Korean NeurosurgSoc 48: $298-300$

6. Hadden RD, Cornblath DR, Hughes RA, Zielasek J, Hartung HP, et al. (1998) Electrophysiological classification of Guillain-Barré syndrome: clinical associations and outcome. Plasma Exchange/SandoglobulinGuillain-Barré Syndrome Trial Group. Ann Neurol 44: 780-8. 